\title{
A unified approach to the estimation and interpretation of resistance costs in plants
}

\author{
MM Vila-Aiub ${ }^{1}, \mathrm{P} \mathrm{Neve}^{2}$ and F Roux ${ }^{3}$ \\ ${ }^{1}$ Department of Ecology, IFEVA (CONICET), Universidad de Buenos Aires (UBA), Buenos Aires, Argentina; ${ }^{2}$ School of Life Sciences, \\ University of Warwick, Wellesbourne, Warwickshire, UK and ${ }^{3}$ Laboratoire de Génétique et Evolution des Populations Végétales, \\ FRE-CNRS 3268, Université de Lille 1, Villeneuve d'Ascq Cedex, France
}

\begin{abstract}
Plants exhibit a number of adaptive defence traits that endow resistance to past and current abiotic and biotic stresses. It is generally accepted that these adaptations will incur a cost when plants are not challenged by the stress to which they have become adapted-the so-called 'cost of adaptation'. The need to minimise or account for allelic variation at other fitness-related loci (genetic background control) is frequently overlooked when assessing resistance costs associated with plant defence traits. We provide a synthesis of the various experimental protocols that accomplish this essential requirement. We also differentiate those methods that enable the identification of the trait-specific or
\end{abstract}

Keywords: defence traits; resistance costs; fitness; genetic background

\section{Introduction}

Plants are continuously exposed to a suite of novel and ever-changing abiotic and biotic stresses. Local plant adaptive responses to these stresses may occur through the evolution of defence strategies. Plant defence mechanisms elicit constitutive and inducible traits that prevent or limit damage or mortality (resistance) and/or improve the ability to recover from damage (tolerance) (Restif and Koella, 2004; Núñez-Farfán et al., 2007) (no distinction between resistance and tolerance to stresses will be made in this study unless otherwise stated).

Central to evolutionary biology, population genetics and plant physiology is the principle that novel adaptive defence traits are not cost free (Herms and Mattson, 1992; Bergelson and Purrington, 1996). Evolved resistance alleles, by their nature, endow a fitness advantage over the wild allele in the presence of the stress. However, it is argued that resistance alleles will have pleiotropic fitness costs when the stress factor is removed from the environment-the so-called 'cost of resistance'. Pleiotropic costs of resistance may pose limits on evolution by natural selection and their likely existence is based on observed polymorphism at alleles for pathogen, herbivore and herbicide resistance in natural and agricultural

Correspondence: Dr MM Vila-Aiub, Department of Ecology, IFEVA (CONICET), Universidad de Buenos Aires (UBA), Avenida San Martin 4453-Facultad de Agronomia, Buenos Aires 1417, Argentina.

E-mail: vila@ifeva.edu.ar

Received 6 January 2010; revised 26 November 2010; accepted 24 February 2011; published online 4 May 2011 mechanistic basis of costs (direct methods) from those that provide an estimate of the impact of costs by examining the evolutionary trajectories of resistance allele frequencies at the population level (indirect methods). The advantages and disadvantages for each proposed experimental design are discussed. We conclude that plant resistance systems provide an ideal model to address fundamental questions about the cost of adaptation to stress. We also propose some ways to expand the scope of future studies for further fundamental and applied insight into the significance of adaptation costs. Heredity (2011) 107, 386-394; doi:10.1038/hdy.2011.29; published online 4 May 2011 systems (Fritz and Simms, 1992; Preston and Powles, 2002; Neve and Powles, 2005).

A wealth of studies have sought to test the resistance cost hypothesis for adaptation to herbicide, herbivore and pathogen pressure (see reviews by Bergelson and Purrington, 1996; Coustau et al., 2000; Purrington, 2000; Strauss et al., 2002; Burdon and Thrall, 2003; Vila-Aiub et al., 2009a,b). These studies have acknowledged the importance of resistance costs in predicting the spread of plant resistance alleles and determining their population equilibrium frequencies under a range of environmental conditions. A better understanding of the cost/benefit trade-offs of resistant and susceptible alleles has practical implications for the management of crop resistance to diseases, insects and herbicides in modern agriculture. There are also benefits for transgenic crop technology in understanding the plant fitness consequences of defencerelated resistance alleles (Brown, 2002; Neve, 2007; Green, 2009; Vila-Aiub et al., 2005b, 2009a, b) and for minimising risks of transgene dissemination into the wild flora (Ellstrand et al., 1999).

There are inherent difficulties in measuring the expression and magnitude of costs of resistance/tolerance defence genes, meaning that the choice of experimental design and plant material is critical. Control of genetic background is essential to unequivocally ascribe fitness costs to those genes that endow resistance (Bergelson and Purrington, 1996). By controlling the genetic background, the genetic variability between resistant and susceptible genotypes is restricted, so that only the effect of the allelic substitution at the resistance locus on plant fitness traits is assessed. This applies for 
the effect of traits derived of single allele substitutions at one locus, such as target-site herbicide resistance genes (Yu et al., 2007) or allele replacements in multilocus system-based host-parasite resistance interactions (Thrall and Burdon, 2003). Where control of genetic background is not achieved, it is likely that differential fitness will result from polymorphisms at non-resistance alleles and from other effects caused by inbreeding depression, non-random mating and linkage disequilibrium processes (Marshall and Ellstrand, 1986; Falconer and Mackay, 1996; Keller and Waller, 2002).

Despite its importance, the lack of genetic background control has been a major obstacle to interpretation of studies attempting to define the cost of plant resistance to environmental stresses (Bergelson and Purrington, 1996; Coustau et al., 2000; Strauss et al., 2002; Vila-Aiub et al., $2009 a, b)$. Analyses have revealed that 50 and $25 \%$ of studies evaluating resistance costs associated with herbivory (Strauss et al., 2002) and herbicides (Vila-Aiub et al., 2009a, b), respectively, have not complied with this essential experimental condition.

In order to estimate the expression and magnitude of resistance costs, comparison of fitness traits has been repeatedly carried out by comparing $R$ and $S$ phenotypes from distinct plant populations ('between 2 populations' approach). Although the 'between 2 populations' approach has been the most widely used method found in the last four decades of the literature, it presents the greatest limitations when assessing resistance costs (Bergelson and Purrington, 1996; Strauss et al., 2002; Vila-Aiub et al., 2009a,b). For studies assessing costs associated with herbicide resistance alleles, the 'between 2 populations' approach comprises c. $41 \%$ of the examined literature (Supplementary Table S1). The major and fundamental limitation of this methodology is that putative costs of resistance may be artefacts, as phenotypes originating from different geographical locations or environments will likely have been selected for different fitness traits and this would impede any valid fitness comparison between them in a common environment (Bergelson and Purrington, 1996; Strauss et al., 2002; VilaAiub et al., 2009a, b). Furthermore, inbreeding depression, non-random mating and linkage disequilibrium may also be present in field-collected $R$ and $S$ populations resulting in other confounding effects (Marshall and Ellstrand, 1986; Keller and Waller, 2002).

The motivation for the present contribution is to provide a synthesis of the various experimental designs and techniques available to account for allelic differences at loci other than those conferring resistance and tolerance. In particular, we will focus on those methods that (i) examine resistance costs within a random homogenous genetic background, (ii) quantify the effects of different genetic backgrounds on costs and (iii) eliminate or reduce the effects of genetic background on costs. We will also show that experimental methods can be distinguished on the basis of whether they are able to determine the trait-specific or mechanistic basis of costs (that is direct methods) or whether they provide an estimate of the impact of costs by examining the evolutionary trajectories of resistance allele frequencies at the population level in the absence of selection (that is indirect methods). The advantages and disadvantages for each proposed experimental design are discussed (Table 1).
For the particular case of herbicide resistance alleles in weed species, the extent to which each of the above methods have been used in the published literature is summarised in Table 1 . Ninety-three experimental protocols contained in 90 published studies were identified in citation databases (Scopus (Elsevier, Amsterdam, the Netherlands) and Web of Science (Thomson Reuters, Philadelphia, PA, USA)) (Supplementary Table S1).

Resistance to pathogens and herbicides represents good models to study costs of resistance, as the molecular and the genetic basis of resistance is often known and, therefore, most recent methodological advances have been made in studying these traits. Our review will mainly be illustrated by studies in these research areas. Finally, we propose a number of research areas that require further understanding and evaluation in the context of costs associated with plant adaptive defence traits.

\section{Methodological approaches to control for genetic background}

\section{Direct methods: comparison of traits}

Direct methods enable the identification of the modified plant traits that result in the expression of resistance costs. For instance, the expression of costs associated with the chloroplastic psbA gene mutation (Ser-264-Gly) leading to triazine herbicide resistance in many weed species has been shown to originate from limited electron transfer within the PSII complex. This leads to reduced photosynthesis, lower growth rates, less competitive ability and reduced seed production (reviewed by Holt and Thill, 1994).

The most prevalent experimental design in the literature to assess resistance costs involves the comparison of plant survival and growth traits between resistant $(R)$ and susceptible $(S)$ genotypes (Bergelson and Purrington, 1996; Strauss et al., 2002; Vila-Aiub et al., $2009 a, b)$. Biomass allocation to vegetative and reproductive organs has often been regarded as the 'common currency' to compare relative fitness between $R$ and $S$ genotypes. These comparisons enable the identification of life history stages in which resistance costs are expressed (direct methods), and this knowledge can be useful for the management of resistance in humanrelated activities, such as agriculture (herbicide-resistant weeds and disease resistance in crops).

Assessing costs within a random homogenous genetic background: Discrete $\mathrm{R}$ and $\mathrm{S}$ phenotypes from within single populations: Recently evolved resistance traits will rarely be fixed in plant populations. Therefore, providing that some random mating between $R$ and $S$ phenotypes has taken place under relaxed selection, $R$ and $S$ phenotypes will exist in a homogenous genetic background in a single population. If suitable experimental protocols are available to phenotype $R$ and $S$ individuals within these populations (Vila-Aiub et al., $2005 b)$, then these individuals can be grown to maturity, so that $R$ and $S$ seed can be collected for subsequent experiments.

A number of recent studies evaluating resistance costs associated with target and non-target-site herbicide 
Table 1 Summary of the experimental protocols that enable to account for allelic differences at loci other than those conferring resistance among compared resistant and susceptible plant genotypes

\begin{tabular}{|c|c|c|c|c|c|c|c|c|c|}
\hline \multirow[t]{2}{*}{ Method } & \multicolumn{7}{|c|}{ Direct methods } & \multicolumn{2}{|c|}{ Indirect methods } \\
\hline & $\begin{array}{c}\text { Single } \\
\text { population }\end{array}$ & $\begin{array}{l}\text { Pedigreed } \\
\text { lines }\end{array}$ & $\begin{array}{l}\text { Segregating } \\
\text { population }\end{array}$ & $\begin{array}{c}\text { Multiple } \\
\text { populations }\end{array}$ & $\begin{array}{l}\text { Segregating } \\
\text { cross }\end{array}$ & NILs & $\begin{array}{c}\text { Transgenic } \\
\text { lines }\end{array}$ & $\begin{array}{c}\text { Multi- } \\
\text { generational }\end{array}$ & Cline \\
\hline (a) Prevalence ${ }^{a}$ & 4.5 & 1.0 & 18.2 & 18.2 & 2.0 & 4.3 & 2.0 & 7.5 & 1.0 \\
\hline \multicolumn{10}{|l|}{ (b) Advantages/disadvantages } \\
\hline Mechanistic bases $^{\mathrm{b}}$ & + & + & + & + & + & + & + & - & - \\
\hline Fitness $\cos ^{\mathrm{c}}$ & - & - & - & - & - & - & - & + & + \\
\hline Time consumption (generation) & 2 & 2 & $2-3$ & 0 & $2-3$ & $6-7$ & $3-4^{\mathrm{d}}$ & $5-6$ & 1 \\
\hline Genetic background control ${ }^{\mathrm{e}}$ & + & + & + & ++ & ++ & +++ & +++ & & $\mathrm{f}$ \\
\hline Assumptions & $\begin{array}{l}\text { No } \\
\text { sub-population } \\
\text { structure }\end{array}$ & $\begin{array}{c}\text { Quantitative } \\
\text { traits } \\
\text { only }\end{array}$ & & $\begin{array}{l}\text { No } \\
\text { population } \\
\text { structure }\end{array}$ & & & & & \\
\hline $\begin{array}{l}\text { Genotyping and } \\
\text { phenotyping cost }\end{array}$ & + & $+^{\mathrm{g}}$ & + & + & + & + & + & - & - \\
\hline \multicolumn{10}{|l|}{ (c) Recommendations } \\
\hline Model organism & & & & & & & $\mathrm{x}$ & & $\mathrm{x}$ \\
\hline Selfing species & & $\mathrm{x}$ & $\mathrm{x}$ & & $\mathrm{x}$ & & & & $\mathrm{x}$ \\
\hline Outcrossing species & $\mathrm{x}$ & ? & $\mathrm{x}$ & & $\mathrm{x}$ & & & & $\mathrm{x}$ \\
\hline
\end{tabular}

Abbreviation: NILs, near-isogenic lines.

A number of advantages, disadvantages and recommendations are described for each protocol (for more details see text).

${ }^{a}$ Use $(\%)$ of experimental designs in published studies $(n=93)$ assessing pleiotropic effects associated with herbicide resistance alleles.

$\mathrm{b}^{\prime}+{ }^{\prime}$ ' and ' - ' stand for methods that provide or not an understanding of the particular trait involved in the expression of cost, respectively.

${ }^{\prime}$ ' + ' and ' - ' stand for methods that evaluate or not the impact of costs on the evolutionary trajectories of resistance allele frequencies, respectively.

${ }^{\mathrm{d}}$ It will depend on the reproductive system of the species.

${ }^{\mathrm{e}}$ Methods that examine costs within a random homogenous genetic background (+), provide an understanding of the effects of different genetic backgrounds on costs $(++)$ and eliminate the effects of genetic background $(+++)$.

The genetic background control for the indirect methods depends on the plant material used.

${ }^{\mathrm{g}}$ Difficult to determine resistance genotype.

$\mathrm{h}^{\prime}+{ }^{\prime}$ and ' - ' stand for low and high genotyping and phenotyping cost, respectively.

resistance alleles have employed this technique (VilaAiub et al., 2005a, b; Pedersen et al., 2007; Vila-Aiub et al., 2009a, b). In these studies, vegetative clones of individual plants were propagated and herbicide was applied to a single clone to enable individuals to be phenotyped as herbicide $R$ or $S$. $R$ and $S$ plants were then individually bulk crossed to produce $R$ and $S$ seed populations. We have termed this the 'single population' approach (Vila-Aiub et al., 2005b).

A potential pitfall of the 'single population' protocol is that it is unable to discriminate between heterozygous $(R S)$ - and homozygous $(R R)$-resistant individuals. In the case where $R S$ plants are not associated with resistance costs (that is the cost of resistance is recessive; Roux et al., 2004), an underestimation of resistance costs associated with $R R$ plants may occur. This drawback can be overcome by plant genotyping using allele-specific PCR techniques and thus enabling the identification of $R S$ and $R R$ plants (Délye et al., 2002).

Pedigreed lines: A range of possibilities exist for estimating costs of resistance based on the production of pedigreed seed populations. This method does not require any prior knowledge of the mechanism of resistance and is best suited to estimating costs of resistance traits that are inherited in a quantitative or semi-quantitative fashion. In selfing species, seeds collected from a single plant represent an inbred line. Then, once appropriate seed families (maternal lines) have been produced, this method enables the quantification of costs through the statistical correlation between the resistance level of the plant families or phenotypes and any measured fitness trait.

Baucom and Mauricio (2004) used this approach to determine costs associated with increased glyphosate tolerance in maternal families of Ipomoea purpurea. They collected seed from 32 individual plants (grandmaternal lines), grew five seeds from each of these lines in a common garden and selfed these plants for one generation. Seeds were collected from each selfed plant and seeds from the five plants from each grandmaternal line were bulked to create 32 maternal lines. For each of these lines, mean glyphosate tolerance and mean fitness in the absence of glyphosate was determined. A clear trade-off between fitness costs and benefits was apparent: those individuals with higher glyphosate tolerance expressed the higher resistance costs. Similarly, the 'pedigree lines' approach has also enabled the identification of a tradeoff between reproduction and herbivory defence traits in Arabidopsis thaliana inbred lines: those lines with higher trichome density and glucosinolate concentration have shown an impaired production of fruits (Mauricio, 1998).

In the above studies, allelic differences among different inbred or family lines were not completely accounted for. To reduce the impact of different genetic backgrounds on the expression of resistance costs, intermating among field-collected plants should have been required before production of maternal lines. 
Controlled segregating populations: This protocol requires the formation of a segregating $\mathrm{F}_{2}\left(\right.$ or $\mathrm{F}_{3}$ ) population resulting from either a single-parent mating (that is crossing of multiple paired homozygous $R R$ plants with homozygous SS plants) (Jordan, 1996; Roux et al, 2004; Roux and Reboud, 2005) or from crosses among multiple parents (that is crossing several $R S$ plants) (Menchari et al., 2008). As expected for crosses involving a greater control of genetic background, fitness costs have been more frequently reported in progeny originated from single-parent mating than when using crosses among multiple parents (Bergelson and Purrington, 1996). Despite of this, the use of single-parent mating has limitations, as it will depend on the extent to which costs are expressed in the selected genetic backgrounds.

The various 'segregating population' protocols have been employed in c. $20 \%$ of studies evaluating costs of herbicide resistance alleles (Table 1). For instance, an $\mathrm{F}_{2}$ segregating $A$. thaliana population (single-parent mating approach) has been used to demonstrate a $37 \%$ recessive fitness cost ( $R R$ vs $R S=S S)$ associated with the chlorsulfuron resistance endowing Pro-197-Ser acetolactate synthase mutation (Roux et al., 2004). Production of $F_{2}$ and $F_{3}$ lines has also enabled the assessment of the first documented ecological cost associated with a herbicide resistance allele: increased susceptibility to herbivorous insects associated with a chloroplastic $p s b A$ mutation in D1 protein of photosystem II (Gassmann, 2005).

An important attribute of the 'segregating population' approach is that it may enable the estimation of the dominance of the resistance cost (Carrière et al., 2001; Vacher et al., 2003; Roux et al., 2004), which, despite its importance in determining the initial spread and establishment of resistance alleles in populations, has seldom been estimated in plants (Roux et al., 2004; Menchari et al., 2008). However, the dominance of the cost can only be determined when it is possible to distinguish between $S S, R S$ and $R R$ genotypes in a segregating population by allele-specific PCR.

The control of genetic background effects achieved through the use of the 'segregating population' approach does not, however, completely overcome the linkage disequilibrium around the resistance gene. It is, therefore, possible that fitness costs may result from genetic variation at loci linked to the resistance gene in the two parents and not from the resistance allele itself.

\section{Quantifying the effects of different genetic backgrounds} on costs: Field-collected R and S populations: Comparison of multiple field-collected $R$ and $S$ populations provides some statistical power to account for the effect of differences in genetic background (Cousens et al., 1997; Strauss et al., 2002). This experimental design assumes that a statistically significant difference in mean trait values between compared $R$ and $S$ populations indicates that those differences are likely caused by pleiotropic effects of resistance gene(s) and not as a result of a type I error. This experimental approach was followed by Tardif $e t$ al. (2006), who identified a significant resistance cost correlated with the Trp-574-Leu acetolactate synthase mutation in six Amaranthus powellii populations. The resistance allele conferred pleiotropic effects on plant morphology and anatomy resulting in impaired resource acquisition in a comparison of six field-collected $R$ and six $S$ populations. For a proper comparison to be made, this method requires all resistant genotypes to have identical resistance alleles (that is Trp-574-Leu ) as different alleles may be associated with different resistance costs (Roux et al., 2004; Vila-Aiub et al., 2009a, b).

While the multiple population approach does not control for differences in genetic background between $R$ and $S$ lines, one of its advantages is that helps determine the importance of different genetic backgrounds in influencing the fitness of resistance genes. This experimental protocol has been the second most widely used to estimate costs associated with herbicide resistance alleles (Table 1).

Multiple segregation crosses: Another method that enables the evaluation of the influence of the genetic background on variations in the magnitude and expression of resistance costs consists of pairing either the same resistant line (RR or resistant) with different SS (or susceptible) plants collected from different environments (locations, regions and so on) (Paris et al., 2008) or multiple $R S$ plants for a particular resistance allele, but originated from different populations (Menchari et al., 2008). By analysing the same 2,4-D herbicide resistance mutation in eight different segregating genetic backgrounds, Paris et al. (2008) report that the fitness cost of a resistance gene is variable depending on the local genetic composition. The study provides evidence for the existence of compensatory genes that are segregating in different genetic backgrounds (see below Compensatory evolution of resistance costs).

The multiple segregation crosses method has seldom been used in the literature, although its significant power to detect the variable effect of genetic background on resistance costs (Table 1).

Eliminating the effects of genetic background on costs: Introgression of resistance allele into a susceptible background: backcross (near-isogenic lines): Isogenic lines, in which resistance alleles are expressed in a susceptible background, can be produced by crossing a single $R$ and $S$ plant followed by repeated backcrossing of $R$ progeny into the $S$ background. Introgression of the $R$ allele is completed by selfing or crossing the resulting $R S$ plants, in order to produce homozygote $R R$ plants (the so-called 'near-isogenic lines (NILs)'), whose fitness is compared with the maternal SS line. It is rarely possible to produce lines that are entirely isogenic at all loci other than those conferring resistance. The production of 'NILs' aims to produce $R R$ plants having a maximum percentage of genes of the susceptible genetic background. The degree to which this can be achieved is correlated with the number of generations of backcross.

Despite its ability to unambiguously identify resistance costs (Bergelson and Purrington, 1996), the NILs protocol has rarely been used (pathogen resistance: Heidel et al., 2004; Korves and Bergelson, 2004; Laughlin et al., 2009; herbicide resistance: McCloskey and Holt, 1990, 1991; Salzmann et al., 2008; Wang et al., 2010) (Table 1; Supplementary Table S1). Several plant generations are required to produce NILs in order to disrupt linkage disequilibrium between resistance and other genes. If, however, polymorphisms physically related to the resistance gene are still present after several $R S \times S S$ backcrosses, then these gene linkages may be 
thought as genuine causes of resistance costs (Strauss et al., 2002).

Introgression of resistance allele into a susceptible background: transgenic plants: The classical transgenic approach involves the introduction of a resistance gene via a vector into a susceptible genetic background in order to obtain plants whose genomes differ solely due to the presence of the studied resistance gene. Transgenic lines are usually backcrossed with the $S$ wild type to obtain homozygous lines with and without the transgene. Any fitness differences between $S$ and transgenic $(R)$ lines may not, however, be entirely due to the effect of the resistance gene. The introduction of foreign DNA may modify the expression of the genome near the site of insertion and this may have fitness implications unrelated to expression of the resistance allele. Thus, several independent transgenic lines have to be generated to account for these positional effects (Bergelson et al., 1996). Inclusion of marker genes, such as antibiotic resistance, as part of the transgene makes it possible to identify plants that have been successfully transformed. However, these linked marker genes may also impose their own pleiotropic fitness costs.

A small number of published studies have generated resistant transgenic plants and these studies have demonstrated the enormous potential of this technique for assessing resistance costs in a completely controlled genetic background (Bergelson et al., 1996; Purrington and Bergelson, 1997, 1999; Burke and Rieseberg, 2003; Jackson et al., 2004; Al-Ahmad et al., 2005, 2006; AlAhmad and Gressel, 2006). After successfully controlling the effect of the vector plasmid and insertion position, Bergelson et al. (1996) demonstrated the expression of environmentally dependent negative pleiotropic effects on fecundity associated with the Pro-197-Ser acetolactate synthase herbicide resistance mutation in $A$. thaliana. Similarly, transgenic $A$. thaliana lines overexpressing cold resistance genes $\left(\mathrm{CBF}_{2-3}\right)$ have been shown to exhibit costs in cold-free environments (Jackson et al., 2004).

The Cre-lox method makes it possible to eliminate the positional effect of the transgene on resistance cost expression (Tian et al., 2003). The creation of Cre-lox lines has been described in detail elsewhere (Bayley et al., 1992; Osborne et al., 1995). Briefly, Cre-lox lines enable the insertion of resistance alleles into $S$ backgrounds in constructs, where they are flanked by DNA sequences that enable subsequent recombinational excision of the $R$ allele to produce $S S$ and $R R$ Cre-lox isolines. The Cre-lox protocol allows measurement of the fitness costs of the resistance gene only by comparison of individuals with the vector and transgene $(R R)$ with individuals with the vector (inserted in the identical position), but without the transgene (SS). To date, the Cre-lox method has been used only once and has enabled the identification of a resistance cost associated with the pathogen resistance gene RPM1 in A. thaliana in the absence of pathogens (Tian et al., 2003).

\section{Indirect methods: dynamics of resistance allele}

\section{frequencies}

Resistance costs may express in some but not all plant life history stages (Boege et al., 2007; Vila-Aiub et al., $2009 a, b)$. As a result, pleiotropic effects of resistance genes should ideally be examined across all plant life history stages. A general approach that achieves this goal is the study of herbicide resistance allele frequencies in isolated or interconnected populations over a number of generations. This method relies on the expectation that a costly resistance allele will decrease in frequency over time, so that significant deviations of observed from expected resistance frequencies (allelic/genotypic/phenotypic) under no herbicide selection provides clear evidence for the expression and magnitude of resistance costs.

A limitation of indirect methods is that they require the genotyping (if the molecular genetic basis of resistance is known) or phenotyping of several hundreds or thousands of individuals in order to ascertain changes in resistance frequency. Where the allelic basis of resistance is known, the availability of high throughput genotyping methods makes these approaches possible (Giancola et al., 2006).

As for direct methods, the validity of the results obtained from these approaches depends on proper control of genetic background. Unlike 'direct methods', these approaches do not allow the identification of the particular plant traits involved in the expression of costs of resistance. Despite its enormous power to detect resistance costs in insects and pathogens (Cochran, 1993; Bahri et al., 2009; Li et al., 2009), this experimental protocol has not proved to be popular in published literature to date in plants (Table 1; Supplementary Table S1).

Multigenerational study: This protocol assesses resistance frequencies over multiple discrete, isolated (no migration) and non-overlapping generations. For detecting relatively small resistance costs, the protocol requires large population sizes, and several replicates and/or plant generations to overcome the stochastic effects of genetic drift. Depending on the plant material used (segregating populations, NILs and so on), it may also be necessary to employ molecular techniques for genotyping analysis.

Using $\mathrm{F}_{2}$ segregating populations, Roux et al. (2005) studied allele frequencies of acetolactate synthase, cellulose synthase and auxin-induced target-site resistance genes over seven $A$. thaliana generations under controlled environmental conditions. Clear deviations of observed from expected resistance allele frequencies (with the assumption of no fitness cost) for some but not all resistance alleles enabled the assessment of corresponding resistance costs, which varied from 0 to 94\% (Roux et al., 2005). Other studies that have used this approach under field conditions (using $\mathrm{F}_{2}$ segregating populations ('segregating population') or discrete $S$ and $R$ phenotypes ('single population')) have successfully identified significant resistance costs associated with glyphosate (Preston et al., 2009) and P450-based enhanced herbicide metabolism endowing resistance alleles (Vila-Aiub, Neve and Powles, unpublished).

Cline and migration-selection balance: In natural populations located along an ecological gradient, a gradient of allele frequency (that is, cline) is expected for the genes selected by the corresponding ecological factor (Nagylaki, 1975). In the case of resistance, the shape of the cline step depends on the fitness difference 
among the $S S, R S$ and $R R$ genotypes in the regions with the selective pressure and the regions without the selective pressure, as well as the amount of gene flow between the two types of regions (Lenormand et al., 1999; Lenormand and Raymond, 2000; Labbé et al., 2009).

By experimentally fixing the migration rate among experimental populations, Roux et al. (2006) built up an artificial cline for estimating the resistance costs associated with csr1-1 (Pro-197-Ser) acetolactate synthase and axr2-1 (Pro-87-Ser) AXR2 herbicide resistance alleles in A. thaliana. The evolution of the resistance allele frequency was estimated along a transect across treated and untreated areas over discrete generations. Only three generations were sufficient to precisely estimate the resistance cost of those two herbicides resistance alleles: 10 and $92 \%$ for the $\operatorname{csr} 1-1$ and axr2-1 alleles, respectively. In contrast with isolated populations in multigenerational studies, control of gene flow among populations also allows the effect of genetic drift on resistance cost estimates to be reduced. This point has been illustrated for the csr1-1 resistance allele. While the use of 12 isolated populations (each of 120 plants) precluded the detection of costs lower than 15\% (Roux et al., 2005), adding gene flow among experimental populations (each of 100 plants) allowed estimation of a fitness cost of $10 \%$ for the csr1-1 allele (Roux et al., 2006).

\section{Future research}

To this point we have reviewed and evaluated the various methods available for control of genetic background in order to perform studies to estimate costs of evolved defence mechanisms in plants. Most previous studies, many which have used flawed methodologies, have been primarily concerned with estimating the presence and extent of these costs either to explain the maintenance of polymorphism for resistance traits within plant populations or to assess the potential for costs of resistance to moderate the rate of resistance evolution in agricultural settings. Plant resistance systems provide an ideal model to address fundamental questions about the cost of adaptation, particularly as, in many cases, the molecular genetic basis of resistance is known. In the remaining sections, we propose some ways in which the scope of these studies can be expanded to provide further fundamental and applied insight into the significance of these costs of adaptation. Four relevant research areas are proposed below.

\section{Genome-wide control of false positives when using natural populations}

In the area of genome-wide association scans, it is well known that spurious genetic marker-trait associations may arise from confounding population structure, leading to a high false-positive rate (Lander and Schork, 1994). When using natural plant material for estimating resistance fitness costs, similar effects may confound results when $R$ and $S$ plants originate from different plant populations (that is 'between 2 populations' and 'multiple populations' methods). Moreover, population structure can also lead to a biased estimation of the effect of the gene under study. For example, a mutation in the $V g t 1$ flowering time gene was initially found to explain
$17 \%$ of the phenotypic variation in flowering time in maize (Ducrocq et al., 2008). When population structure was taken into account, this percentage decreased to $4 \%$. So, $13 \%$ of the flowering time variation was accounted for by population structure. We believe that there is an urgent need for estimating genetic relatedness among individuals phenotyped for resistance using genetic markers distributed across the genome. Those genetic relatedness estimates may then be integrated in appropriate statistical methods for reducing the false-positive rate and adequately estimating the additive effect of the resistance gene (Kang et al., 2008).

\section{Compensatory evolution of resistance costs}

A number of experimental protocols have been proposed to enable more meaningful evaluations of resistance costs and many of these focus on removing the confounding effects of genetic background; however, it should be recognised that resistance alleles are expressed in a range of genetic backgrounds in natural populations. Significant effects of dissimilar genetic backgrounds on the expression and magnitude of several resistance mutations have been documented (Bergelson, 1994; Bergelson and Purrington, 1996; Menchari et al., 2008; Paris et al., 2008). Given this, we may easily speculate that pleiotropic resistance costs may be compensated by natural selection at other polymorphic loci within the genome (Maisnier-Patin and Andersson, 2004). This process creates the opportunity for co-adaptation or integration of the new resistance allele into the genome (Cohan et al., 1994; Guillemaud et al., 1998). By crossing an herbicide-resistant line (axr1-3 resistance allele) to nine natural accessions, Paris et al. (2008) showed a potential compensation of both the resistance cost and its associated dominance by the genetic diversity present within $A$. thaliana. To our knowledge, no study has been specifically designed to detect the genetic basis of resistance cost modifiers in plants. Given the common and quick development of molecular markers in plant species, quantitative trait loci mapping or genome-wide association mapping might be used to find the genetic basis of compensation (Nordborg and Weigel, 2008).

\section{Dominance and frequency dependence of resistance costs}

Dominance and frequency dependence both influence the expression of resistance costs and thus the evolutionary dynamics of the corresponding resistance genes. Little attention has been given to understanding these effects and further research is required. The adaptive value of newly arisen herbicide resistance alleles depends on the dominance of the fitness cost. For resistance endowed by major genes, the first evolved resistant mutants in populations will be heterozygous $(R S)$. Thus, an understanding of the effect of the number of allele copies on the expression of costs is pivotal. Given the very few studies designed to address this question, it is not possible to discern a general pattern. The very few relevant studies have shown that most resistance costs associated with herbicide resistance alleles are recessive, an indication that few constraints to the initial selection of resistance alleles exist (Roux et al., 2004; Menchari et al., 2008). 
The magnitude of resistance costs may also be dependent on the frequency of resistance alleles in populations. If costs are less evident when the resistance frequency is relatively small (negative frequency dependence), then the resistance allele frequency prior to selection will be higher than if costs are positively frequency dependent. While the axr2-1 herbicide resistance mutation has been shown to be associated with higher fitness costs when its frequency increased (Roux et al., 2006), the cost of ixr1-2 herbicide resistance mutation increased with decreased resistance frequency (Roux et al. 2005). Frequency-dependent changes in resistance costs may result from density-dependent changes in the intensity and importance of competitive interactions among individuals carrying resistance alleles (Vila-Aiub et al., 2009a, b).

\section{Expression of resistance costs in the presence of the selective agent}

Usually, it is assumed that costs of resistance will only be detected in the absence of the selective agent. Korves and Bergelson (2004) found a net cost of resistance under pathogen pressure in the A. thaliana-Pseudomonas syringae pathosystem. Pathogen-resistant plants demonstrated a resistance cost when exposed to low pathogen pressure. This result might be explained by infection cost-free developmental response to infection in susceptible plants, or by an inappropriate triggering of defence mechanisms in resistant plants, so that the benefits of resistance are outweighed by the cost at low infection levels. This phenomenon should be investigated in other resistance systems. Since the intensity of the selective pressure is heterogeneous within natural populations (Goss and Bergelson, 2007), it will be interesting to observe if there is a benefit-cost trade-off of a resistance allele under a gradient of selective pressure (Barrett et al., 2009).

\section{Final remarks}

A number of methodological protocols exist to control for the confounding effects of genetic background when assessing plant resistance costs. Advantages and disadvantages exist for all protocols, and the final decision on which to use will depend on available plant material, study objectives, knowledge of the molecular basis of resistance and laboratory facilities. We encourage careful consideration of the most appropriate and meaningful protocol in all studies attempting to measure plant resistance costs.

Estimation of resistance costs is the initial step for the identification of constraints to evolution and fixation of novel adaptive alleles in plants. However, and as reviewed above, quantitative changes in plant costs as a result of genetic compensatory effects, dominance, plant frequency and intensity of the selective factor are possible and need to be addressed by further research efforts to comprehend the dynamics of resistance alleles in natural populations and agroecosystems.

\section{Conflict of interest}

The authors declare no conflict of interest.

\section{References}

Al-Ahmad H, Dwyer J, Moloney M, Gressel J (2006). Mitigation of establishment of Brassica napus transgenes in volunteers using a tandem construct containing a selectively unfit gene. Plant Biotechnol J 4: 7-21.

Al-Ahmad H, Galili S, Gressel J (2005). Poor competitive fitness of transgenically mitigated tobacco in competition with the wild type in a replacement series. Planta 222: 372-385.

Al-Ahmad H, Gressel J (2006). Mitigation using a tandem construct containing a selectively unfit gene precludes establishment of Brassica napus transgenes in hybrids and backcrosses with weedy Brassica rapa. Plant Biotechnol J 4: 23-33.

Bahri B, Kaltz O, Leconte M, De Vallavieille-Pope C, Enjalbert J (2009). Tracking costs of virulence in natural populations of the wheat pathogen, Puccinia striiformis f.sp.tritici. BMC Evol Biol 9: 26.

Barrett LG, Kniskern JM, Bodenhausen N, Zhang W, Bergelson J (2009). Continua of specificity and virulence in plant hostpathogen interactions: causes and consequences. New Phytol 183: 513-529.

Baucom RS, Mauricio R (2004). Fitness costs and benefits of novel herbicide tolerance in a noxious weed. Proc Natl Acad Sci USA 101: 13386-13390.

Bayley CC, Morgan M, Dale EC, Ow DW (1992). Exchange of gene activity in transgenic plants catalyzed by the Cre-lox site-specific recombination system. Plant Mol Biol 18: 353-361.

Bergelson J (1994). The effects of genotype and the environment on costs of resistance in lettuce. Am Nat 143: 349-359.

Bergelson J, Purrington CB (1996). Surveying patterns in the cost of resistance in plants. Am Nat 148: 536-558.

Bergelson J, Purrington CB, Palm CJ, Lopez-Gutierrez JC (1996). Costs of resistance: a test using transgenic Arabidopsis thaliana. Proc R Soc Lond Ser B-Biol Sci 263: 1659-1663.

Boege K, Dirzo R, Siemens D, Brown P (2007). Ontogenetic switches from plant resistance to tolerance: minimizing costs with age? Ecol Lett 10: 177-187.

Brown JKM (2002). Yield penalties of disease resistance in crops. Curr Opin Plant Biol 5: 339-344.

Burdon JJ, Thrall PH (2003). The fitness costs to plants of resistance to pathogens. Genome Biol 4: 227.

Burke JM, Rieseberg LH (2003). Fitness effects of transgenic disease resistance in sunflowers. Science 300: 1250.

Carrière Y, Ellers-Kirk C, Liu YB, Sims MA, Patin AL, Dennehy TJ et al. (2001). Fitness costs and maternal effects associated with resistance to transgenic cotton in the pink bollworm (Lepidoptera: Gelechiidae). J Econ Entomol 94: 1571-1576.

Cochran DG (1993). Decline of pyrethroid resistance in the absence of selection pressure in a population of German cockroaches (Dictyoptera: Blattellidae). J Econ Entomol 86: 1639-1644.

Cohan FM, King EC, Zawadzki P (1994). Amelioration of the deleterious pleiotropic effects of an adaptive mutation in Bacillus subtilis. Evolution 48: 81-95.

Cousens RD, Gill GS, Speijers EJ (1997). Comment: number of sample populations required to determine the effects of herbicide resistance on plant growth and fitness. Weed Res 37: $1-4$.

Coustau C, Chevillon C, ffrench-Constant R (2000). Resistance to xenobiotics and parasites: can we count the cost? Trends Ecol Evol 15: 378-383.

Délye C, Matejicek A, Gasquez J (2002). PCR-based detection of resistance to acetyl-CoA carboxylase-inhibiting herbicides in black-grass (Alopecurus myosuroides Huds) and ryegrass (Lolium rigidum Gaud). Pest Manag Sci 58: 474-478.

Ducrocq S, Madur D, Veyrieras JB, Camus-Kulandaivelu L, Kloiber-Maitz M, Presterl T et al. (2008). Key impact of Vgt1 on flowering time adaptation in maize: evidence 
from association mapping and ecogeographical information. Genetics 178: 2433-2437.

Ellstrand NC, Prentice HC, Hancock JF (1999). Gene flow and introgression from domesticated plants into their wild relatives. Annu Rev Ecol Syst 30: 539-563.

Falconer DS, Mackay TFS (1996). Introduction to Quantitative Genetics, 4th edn. Longman: Essex.

Fritz RS, Simms EL (1992). Plant Resistance to Herbivores and Pathogens. University of Chicago Press: Chicago, USA.

Gassmann AJ (2005). Resistance to herbicide and susceptibility to herbivores: environmental variation in the magnitude of an ecological trade-off. Oecologia 145: 575-585.

Giancola S, McKhann HI, Bérard A, Camilleri C, Durand S, Libeau P et al. (2006). Utilization of the three high-throughput SNP genotyping methods, the GOOD assay, Amplifluor and TaqMan, in diploid and polyploid plants. Theor Appl Genet 112: $1115-1124$.

Goss EM, Bergelson J (2007). Fitness consequences of infection of Arabidopsis thaliana with its natural bacterial pathogen Pseudomonas viridiflava. Oecologia 152: 71-81.

Green JM (2009). Evolution of glyphosate-resistant crop technology. Weed Sci 57: 108-117.

Guillemaud T, Lenormand T, Bourguet D, Chevillon C, Pasteur N, Raymond M (1998). Evolution of resistance in Culex pipiens: allele replacement and changing environment. Evolution 52: 443-453.

Heidel AJ, Clarke JD, Antonovics J, Dong X (2004). Fitness costs of mutations affecting the systemic acquired resistance pathway in Arabidopsis thaliana. Genetics 168: 2197-2206.

Herms DA, Mattson WJ (1992). The dilemma of plants-to grow or defend. Q Rev Biol 67: 283-335.

Holt JS, Thill DC (1994). Growth and productivity of resistant plants. In: Powles SB and Holtum JAM (eds). Herbicide Resistance in Plants. Biology and Biochemistry. Lewis Publishers: Boca Raton, pp 299-316.

Jackson MW, Stinchcombe JR, Korves TM, Schmitt J (2004). Costs and benefits of cold tolerance in transgenic Arabidopsis thaliana. Mol Ecol 13: 3609-3615.

Jordan N (1996). Effects of the triazine-resistance mutation on fitness in Amaranthus hybridus (Smooth Pigweed). J Appl Ecol 33: $141-150$.

Kang HM, Zaitlen NA, Wade CM, Kirby A, Heckerman D, Daly MJ et al. (2008). Efficient control of population structure in model organism association mapping. Genetics $\mathbf{1 7 8}$ 1709-1723.

Keller LF, Waller DM (2002). Inbreeding effects in wild populations. Trends Ecol Evol 17: 230-241.

Korves T, Bergelson J (2004). A novel cost of $\mathrm{R}$ gene resistance in the presence of disease. Am Nat 163: 489-504.

Labbé P, Sidos N, Raymond M, Lenormand T (2009). Resistance gene replacement in the mosquito Culex pipiens: fitness estimation from long-term cline series. Genetics 182: 303-312.

Lander ES, Schork NJ (1994). Genetic dissection of complex traits. Science 265: 2037.

Laughlin KD, Power AG, Snow AA, Spencer LJ (2009). Risk assessment of genetically engineered crops: fitness effects of virus-resistance transgenes in wild Cucurbita pepo. Ecol Appl 19: 1091-1101.

Lenormand T, Bourguet D, Guillemaud T, Raymond M (1999). Tracking the evolution of insecticide resistance in the mosquito Culex pipiens. Nature 400: 861-864.

Lenormand T, Raymond M (2000). Analysis of clines with variable selection and variable migration. Am Nat 155: 70-82.

Li A, Lohmeyer KH, Miller JA (2009). Dynamics and mechanisms of permethrin resistance in a field population of the horn fly, Haematobia irritans irritans. Insect Sci 16: 175-184.

Maisnier-Patin S, Andersson DI (2004). Adaptation to the deleterious effects of antimicrobial drug resistance mutations by compensatory evolution. Res Microbiol 155: 360-369.

Marshall DL, Ellstrand NC (1986). Sexual selection in Raphanus sativus: experimental data on nonrandom fertilization, maternal choice, and consequences of multiple paternity. Am Nat 127: 446.

Mauricio R (1998). Costs of resistance to natural enemies in field populations of the annual plant Arabidopsis thaliana. Am Nat 151: 20-28.

McCloskey WB, Holt JS (1990). Triazine resistance in Senecio vulgaris parental and nearly isonuclear backcrossed biotypes is correlated with reduced productivity. Plant Physiol 92: 954-962.

McCloskey WB, Holt JS (1991). Effect of growth temperature on biomass production of nearly isonuclear triazine-resistant and-susceptible common groundsel (Senecio vulgaris L). Plant Cell Environ 14: 699-705.

Menchari Y, Chauvel B, Darmency H, Delye C (2008). Fitness costs associated with three mutant acetyl-coenzyme A carboxylase alleles endowing herbicide resistance in blackgrass Alopecurus myosuroides. J Appl Ecol 45: 939-947.

Nagylaki T (1975). Conditions for the existence of clines. Genetics 80: 595-615.

Neve P (2007). Challenges for herbicide resistance evolution and management: 50 Years after Harper. Weed Res 47: 365-369.

Neve P, Powles S (2005). High survival frequencies at low herbicide use rates in populations of Lolium rigidum result in rapid evolution of herbicide resistance. Heredity 95: 485-492.

Nordborg M, Weigel D (2008). Next-generation genetics in plants. Nature 456: 720-723.

Núñez-Farfán J, Fornoni J, Valverde PL (2007). The evolution of resistance and tolerance to herbivores. Annu Rev Ecol Syst 38: $541-566$.

Osborne BI, Wirtz U, Baker B (1995). A system for insertional mutagenesis and chromosomal rearrangement using the Ds transposon and Cre-lox. Plant J 7: 687-701.

Paris M, Roux F, Berard A, Reboud X (2008). The effects of the genetic background on herbicide resistance fitness cost and its associated dominance in Arabidopsis thaliana. Heredity 101 499-506.

Pedersen BP, Neve P, Andreasen C, Powles SB (2007). Ecological fitness of a glyphosate-resistant Lolium rigidum population: growth and seed production along a competition gradient. Basic Appl Ecol 8: 258-268.

Preston C, Powles SB (2002). Evolution of herbicide resistance in weeds: initial frequency of target site-based resistance to acetolactate synthase-inhibiting herbicides in Lolium rigidum. Heredity 88: 8-13.

Preston C, Wakelin AM, Dolman FC, Bostamam Y, Boutsalis P (2009). A decade of glyphosate-resistant Lolium around the world: mechanisms, genes, fitness, and agronomic management. Weed Sci 57: 435-441.

Purrington CB (2000). Costs of resistance. Curr Opin Plant Biol 3: 305-308.

Purrington CB, Bergelson J (1997). Fitness consequences of genetically engineered herbicide and antibiotic resistance in Arabidopsis thaliana. Genetics 145: 807-814.

Purrington CB, Bergelson J (1999). Exploring the physiological basis of costs of herbicide resistance in Arabidopsis thaliana. Am Nat 154: S82-S91.

Restif O, Koella J (2004). Concurrent evolution of resistance and tolerance to pathogens. Am Nat 164: E90-E102.

Roux F, Camilleri C, Bérard A, Reboud X (2005). Multigenerational versus single generation studies to estimate herbicide resistance fitness cost in Arabidopsis thaliana. Evolution 59: 2264-2269.

Roux F, Gasquez J, Reboud X (2004). The dominance of the herbicide resistance cost in several Arabidopsis thaliana mutant lines. Genetics 166: 449-460.

Roux F, Giancola S, Durand S, Reboud X (2006). Building of an experimental cline with Arabidopsis thaliana to estimate herbicide fitness cost. Genetics 173: 1023-1031.

Roux F, Reboud X (2005). Is the cost of herbicide resistance expressed in the breakdown of the relationships between 
characters? A case study using synthetic-auxin-resistant Arabidopsis thaliana mutants. Genet Res 85: 101-110.

Salzmann D, Handley RJ, Mueller-Scharer H (2008). Functional significance of triazine-herbicide resistance in defence of Senecio vulgaris against a rust fungus. Basic Appl Ecol 9: 577-587.

Strauss SY, Rudgers JA, Lau JA, Irwin RE (2002). Direct and ecological costs of resistance to herbivory. Trends Ecol Evol 17: 278-285.

Tardif FJ, Rajcan I, Costea M (2006). A mutation in the herbicide target site acetohydroxyacid synthase produces morphological and structural alterations and reduces fitness in Amaranthus powellii. New Phytol 169: 251-264.

Thrall PH, Burdon JJ (2003). Evolution of virulence in a plant host-pathogen metapopulation. Science 299: 1735-1737.

Tian D, Traw MB, Chen JQ, Kreitman M, Bergelson J (2003). Fitness costs of R-gene-mediated resistance in Arabidopsis thaliana. Nature 423: 74-77.

Vacher C, Bourguet D, Rousset F, Chevillon C, Hochberg ME (2003). Modelling the spatial configuration of refuges for a sustainable control of pests: a case study of Bt cotton. J Evol Biol 16: 378-387.
Vila-Aiub MM, Neve P, Powles SB (2009a). Fitness costs associated with evolved herbicide resistance alleles in plants. New Phytol 184: 751-767.

Vila-Aiub MM, Neve P, Powles SB (2005a). Resistance cost of a cytochrome $\mathrm{P} 450$ herbicide metabolism mechanism but not an ACCase target site mutation in a multiple resistant Lolium rigidum population. New Phytol 167: 787-796.

Vila-Aiub MM, Neve P, Powles SB (2009b). Evidence for an ecological cost of enhanced herbicide metabolism in Lolium rigidum. J Ecol 97: 772-780.

Vila-Aiub MM, Neve P, Steadman KJ, Powles SB (2005b). Ecological fitness of a multiple herbicide-resistant Lolium rigidum population: dynamics of seed germination and seedling emergence of resistant and susceptible phenotypes. J Appl Ecol 42: 288-298.

Wang T, Picard JC, Tian X, Darmency H (2010). A herbicideresistant ACCase 1781 Setaria mutant shows higher fitness than wild type. Heredity 105: 394-400.

Yu Q, Collavo A, Zheng MQ, Owen M, Sattin M, Powles SB (2007). Diversity of acetyl-coenzyme a carboxylase mutations in resistant Lolium populations: evaluation using clethodim. Plant Physiol 145: 547-558.

Supplementary Information accompanies the paper on Heredity website (http://www.nature.com/hdy) 